\title{
Electrical Activity of the Bladder Is Attenuated by Intravesical Inhibition of P2X2/3 Receptors During Micturition in Female Rats
}

\author{
Betsy H. Salazar ${ }^{1}$, Kristopher A. Hoffman ${ }^{1}$, Chuan Zhang ${ }^{1,2}$, Alex Kavanagh ${ }^{3}$, Yingchun Zhang ${ }^{1,2}$, Timothy B. Boone ${ }^{1,3}$, \\ Alvaro Munoz ${ }^{1,3, *}$ \\ ${ }^{1}$ Regenerative Medicine and Tissue Engineering Program-Urology, Houston Methodist Research Institute, Houston, TX, USA \\ ${ }^{2}$ Department of Biomedical Engineering, University of Houston, Houston, TX, USA \\ ${ }^{3}$ Department of Urology, Houston Methodist Hospital, Houston, TX, USA
}

Purpose: To simultaneously monitor electrical discharges in various bladder regions and the external urethral sphincter (EUS) during voiding contractions, and to assess the functional role of myogenic modulation of the lower urinary tract (LUT) by ionotropic purinergic receptors containing the $\mathrm{P} 2 \mathrm{X} 3$ subunit.

Methods: Female Sprague-Dawley rats were anesthetized with urethane, and implanted with a suprapubic catheter for open cystometry. Flexible microelectrodes were placed ventrally in the bladder dome, upper bladder, lower bladder, and bladder base, along with the middle section of the exposed EUS. Intravesical P2X3-containing receptors were blocked with AF-323, a specific $\mathrm{P} 2 \mathrm{X} 3-\mathrm{P} 2 \mathrm{X} 2 / 3$ receptor antagonist. A digital electrophysiology amplifier was used to record electrical and cystometric signals throughout the LUT.

Results: Electrical activity in the LUT started before effective voiding contractions. Bladder pressure and electrical waveforms showed consistent out-of-phase activity when compared with the recordings made at the EUS. This pattern was also observed during voiding contractions in the presence of AF-353, supporting the hypothesis that during bladder distension, activation of $\mathrm{P} 2 \mathrm{X} 3$-containing receptors is required for voiding contractions. Furthermore, the inhibition of P2X3-containing receptors significantly decreased the amplitude of electrical signals in the urinary bladder, but not the base or EUS.

Conclusions: Our results provide novel information about the regulation of the micturition process by P2X3-containing receptors located in the inner layers of the bladder.

Keywords: Lower Urinary Tract Symptoms; Urination; Purinergic P2X Receptor Antagonists; Urinary Bladder; Electrophysiology

- Grant/Fund Support: This study was supported by the Brown Foundation and the Houston Methodist Foundation.

- Research Ethics: Procedures were approved by the Institutional Animal Care and Use Committee (IACUC) (ID \# AUP-0615-0044/IS00001252)

at the Houston Methodist Research Institute, and performed in accordance with the guidelines of the National Institutes of Health on the care and use of laboratory animals.

- Conflict of Interest: No potential conflict of interest relevant to this article was reported.

\section{- HIGHLIGHTS}

- A recording system was developed for simultaneous cystometric and electromyographic evaluations in the rat lower urinary tract.

- Antagonists of $\mathrm{P} 2 \mathrm{X} 3$ receptors decrease voiding electrical signals in the detrusor but not striated muscles.

- Bladder P2X3 receptors influence micturition in female rats.

Corresponding author: Alvaro Munoz (D) https://orcid.org/0000-0002-5726-0810 Regenerative Medicine and Tissue Engineering Program-Urology, Houston Methodist Research Institute, 6670 Bertner Ave. R10-114, Houston, TX 77030, USA *Current affiliation: Instituto de Neurociencias Traslacionales, Universidad de Guadalajara, Av. Sierra Mojada 950, Guadalajara, Jalisco, CP 44340, Mexico E-mail: alvaro_munoz@hotmail.com / Tel: +52-33-1058-5200 (Ext. 33679) / Fax: +1713-441-6463

Submitted: October 11, 2017 / Accepted after revision: November 27, 2017
This is an Open Access article distributed under the terms of the Creative Commons Attribution Non-Commercial License (http://creativecommons.org/licenses/by-nc/4.0/) which permits unrestricted non-commercial use, distribution, and reproduction in any medium, provided the original work is properly cited. 


\section{INTRODUCTION}

Micturition is an exceptionally complex and coordinated physiological event involving muscle activity in the lower urinary tract (LUT) with a distinct activation pattern controlled by both autonomic and somatic neuronal pathways [1]. The sensation of bladder filling depends on the activation of different mechanosensitive receptors [2], including ionotropic $\mathrm{P} 2 \mathrm{X} 3$-containing receptors (heteromeric [P2X2/3] 3 and homomeric [P2X3]3, abbreviated in this report as $\mathrm{P} 2 \mathrm{X} 3 \mathrm{R}$ ), which are expressed in afferent nerve terminals of the urinary bladder, where they play a key role in bladder afferent transmission to the central nervous system $[1,3]$.

The urothelium-derived excitatory transmitter adenosine triphosphate (ATP) is released from either nerve terminals or urothelial cells during bladder filling to trigger the activation of P2X1 receptors in detrusor cells, stimulating bladder contractions [4]. Similarly, the same ATP release can activate P2X3R in bladder afferent terminals [5], triggering sensory signals that convey information regarding bladder-filling conditions, as well as signals for bladder pain perception. This activation of $\mathrm{P} 2 \mathrm{X} 3 \mathrm{R}$ is critical for initiation of the micturition reflex $[6,7]$.

Efficient voiding requires coordinated contraction of the detrusor with relaxation of the external urethral sphincter (EUS). Elucidating the associated changes in bladder pressure and the contractile activity of the LUT musculature will provide a better understanding of active bladder functions during the voiding phase and the effects of pharmacological treatments [8-11]. Currently, cystometric and electromyographic evaluations are commonly used to determine real-time bladder pressure and EUS activity, respectively [12]. Despite the widespread studies of EUS electrophysiology, to date, no study has thoroughly investigated the simultaneous coordination of electrical signals from the rodent urinary detrusor with concurrent bladder pressure changes and the EUS $[8-11,13,14]$. Interestingly, a urodynamic model incorporating electromyography of the EUS with bladder pressure monitoring has been developed for LUT evaluation using fully awake rats [14]. Similarly, a recent study compared the electromyographic characteristics of the EUS with changes in bladder pressure of mice and rats with and without spinal cord injury leading to LUT dysfunction $[15,16]$. Nevertheless, to the best of our knowledge, no previous study has presented simultaneous recordings of electrical signals in the detrusor and analyzed their associations with changes in bladder pressure and electromyography of the EUS.

For a more complete representation of the electrophysiologi- cal processes in the LUT required for effective micturition, we used a multichannel recording approach, employing flexible electrodes. Proposals have been made to utilize P2X3R as a target to alleviate chronic pain [17] and neurogenic bladder dysfunction in rats with spinal cord injury [18,19], and as a means to investigate cystometric changes in vivo using specific antagonists [20]. Consequently, we evaluated the effects of the intravesical application of the $\mathrm{P} 2 \mathrm{X} 3 \mathrm{R}$ purinergic receptor antagonist AF-353 [17] on EUS and detrusor electrical activity as well as bladder cystometric properties during the generation of intraluminal pressure high frequency oscillations (IPHFOs) [21].

\section{MATERIALS AND METHODS}

Procedures were approved by the Institutional Animal Care and Use Committee (IACUC) (ID \# AUP-0615-0044/IS00001252) at Houston Methodist Research Institute, and performed in accordance with the guidelines of the National Institutes of Health on the care and use of laboratory animals [22]. Every effort was made to minimize the suffering of the animals and the number of animals used, in accordance with IACUC requirements.

\section{Animals and Surgical Procedure}

A total of 8 female Sprague-Dawley rats, 12 weeks of age and weighing 250-300 g, were purchased from Envigo (Research Model Services, Houston, TX, USA), and housed in a pathogen-free environment under a 12-hour light/dark cycle, a controlled temperature of $25^{\circ} \mathrm{C}$, and ad libitum access to food and water in plastic cages containing corn cob bedding. Rats were anesthetized with a single subcutaneous dose of urethane (1.2 $\mathrm{g} / \mathrm{kg}$ ) to maintain responsiveness of the spinal micturition reflex. After reaching the anesthetic plane, an abdominal incision was performed, the abdominal muscles were dissected, and the pubic bone was mechanically separated to expose the entirety of the LUT (i.e., the urinary bladder and EUS). A suprapubic catheter (PE-50) was introduced into the bladder dome, secured with a 6-0 silk suture, and connected to a 3-way valve for both intravesical infusion and bladder pressure monitoring (World Precision Instruments, Sarasota, FL, USA).

\section{Design of Flexible Microelectrodes}

Microelectrodes were assembled with stainless steel springs 1 $\mathrm{cm}$ in length with an external diameter (ID) of either $0.254 \mathrm{~mm}$ or $0.457 \mathrm{~mm}$ (Fig. 1A). One end of the spring was soldered to a 42-AWG microcoaxial cable $10 \mathrm{~cm}$ in length (Digi-Key Elec- 

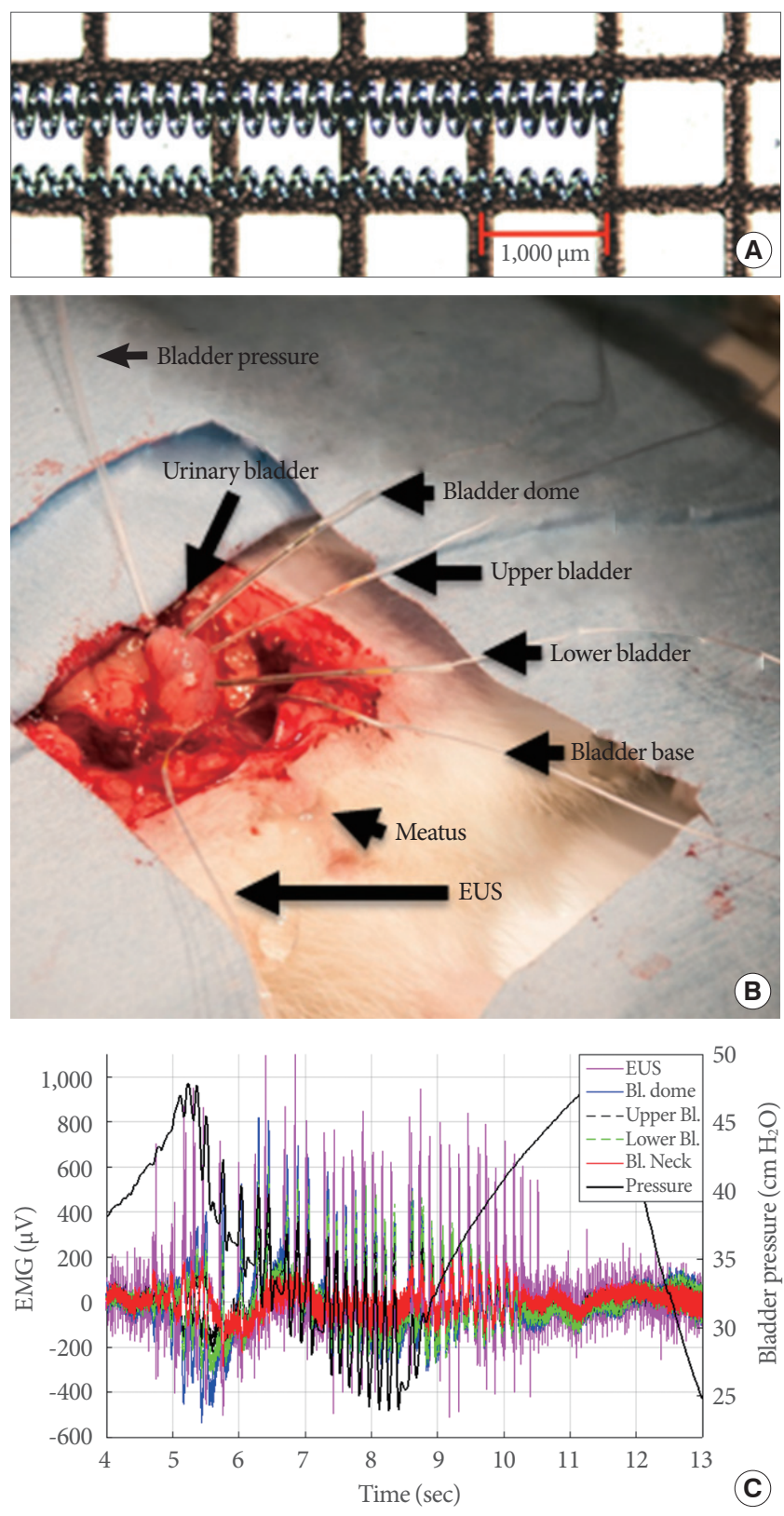

Fig. 1. Recording of electrical signals from the whole lower urinary tract (LUT) during open cystometry. (A) Image of the microelectrodes that were used, with external diameters of 0.457 $\mathrm{mm}$ or $0.254 \mathrm{~mm}$. (B) Photograph of an actual experiment showing the entire LUT and placement of the microelectrodes and the suprapubic catheter for open cystometry. (C) A representative example of simultaneous electrical recordings during a single voiding contraction. EUS, external urethral sphincter; $\mathrm{Bl}$., bladder; EMG, electromyography.

tronics, Thief River Falls, MN, USA), and the solder joint was covered with standard silicone tubing, with an ID of either 0.51 $\mathrm{mm}$ or $0.31 \mathrm{~mm}$. The other end of the microcoaxial cable was fastened to a DuPont-style male crimp pin and receptacle, for connection to the electrode head-stage on the amplifier.

\section{Electromyographic and Cystometric Recording System}

Signals were acquired with a digital electrophysiology amplifier that incorporated both analog and digital filters (RHD2000 Amplifier; Intan Technologies, Los Angeles, CA, USA). Electromyographic signals were recorded with a $5-\mathrm{kHz}$ sampling rate and a bandwidth between $0.09 \mathrm{~Hz}$ and $1 \mathrm{kHz}$. Changes in bladder pressure were recorded using a sampling rate of 1.25 $\mathrm{kHz}$ through an auxiliary port on the amplifier board and in parallel with electromyographic signals. One $0.457-\mathrm{mm}$ electrode was placed on the left pectoral muscle for heart rate monitoring, and 4 more were attached on the bladder dome, upper bladder, lower bladder, and bladder base. Lastly, a $0.254-\mathrm{mm}$ microelectrode was placed in the middle part of the EUS (Fig. 1B). Two additional electrodes were connected to the animal for signal grounding and reference.

\section{Open Cystometric Procedures}

After all electrodes were secured in their positions and the suprapubic catheter was connected to an infusion pump, an intravesical infusion of vehicle solution $(0.9 \% \mathrm{NaCl}+0.01 \%$ dimethyl sulfoxide) was initiated at a perfusion rate of $0.1 \mathrm{~mL} /$ min, as described elsewhere [20]. Recording periods ranged from 30 to 45 minutes for each animal, and contained an average of 10-12 voiding events. Thereafter, the suprapubic catheter was unplugged from the 3-way valve, and the system was flushed with a saline solution containing the P2X3-P2X2/3 antagonist AF-353 [17] at a concentration of $10 \mu \mathrm{M}$ diluted in saline with a final dimethyl sulfoxide concentration of $0.01 \%$. The dose was chosen based on previous research involving the in vivo cystometry of intact Sprague-Dawley rats as well as the contractile response of bladder strips to different concentrations of AF-353 [20]. With the bladder emptied, and the suprapubic catheter reconnected to the infusion pump, perfusion with the P2X3R antagonist was restarted for additional 30-45 minutes of recording. Micturition events were defined as voiding contractions accompanied by vehicle expulsion (visually) and the presence of IPHFOs (during cystometry). Due to the supine position of the animal for electromyography, the voided volume was not recorded. If a contraction was $>5 \mathrm{~cm} \mathrm{H}_{2} \mathrm{O}$ but no expulsion was present, it was considered to be a nonvoiding contraction and was not included in the final electromyography 
analysis [21]. All animals were euthanized with $\mathrm{CO}_{2}$ under deep urethane anesthesia at the end of the evaluations.

\section{Chemicals}

Urethane and sterile-filtered dimethyl sulfoxide were purchased from Sigma-Aldrich (St. Louis, MO, USA). The specific P2X2/3 receptor antagonist AF-353 was donated by Afferent Pharmaceuticals (now part of Merck \& Co., Inc., Kenilworth, NJ, USA).

\section{Data Analysis of Electrical Signals of the LUT During Open Cystometry}

Data files were processed using a combination of customized MATLAB (MathWorks, Natick, MA, USA) and Intan (Intan Technologies, Los Angeles, CA, USA) algorithms. Our analysis suggested that frequencies higher than $20 \mathrm{~Hz}$ produced erroneous spikes in amplitude that were present at all times, regardless of any event during the micturition cycle or intercontractile interval, and therefore considered as noise. We considered a frequency range of $5-20 \mathrm{~Hz}$ to be optimal for characterizing LUT electrical activity. Using the bladder pressure data, we were able to identify the beginning and end points of the IPHFOs (Fig. 1C). Peak analysis was performed with custom MATLAB scripts to determine the amplitude $(\mu \mathrm{Amp})$ and frequency $(\mathrm{Hz})$ of the recorded signals at 3 regions, which we defined as prevoid (i.e., before the IPHFO), void (during the IPHFO), and postvoid (after the IPHFO) activity.

\section{Statistical Analysis}

A total of 8 animals were used in this study. To analyze the statistical differences among electromyographic signal amplitudes, analysis of variance was performed for all groups using multiple comparison followed by the Tukey posttest to correct for the multiple comparison and to determine confidence intervals and significance. Statistical comparisons of cystometric parameters were made using the unpaired Student $t$-test for saline versus AF-353 conditions. In all cases, Prism software (GraphPad Prism, La Jolla, CA, USA) was used. All values are presented as mean \pm standard error of the mean, with P-values $<0.05$ considered to indicate statistical significance.

\section{RESULTS}

\section{LUT Electrical Responses During Open Cystometry and Effects of P2X3R Inhibition}

A detailed analysis of electrical responses in the various bladder regions and the EUS during voiding contractions supports the feasibility of our approach (Fig. 2). During vehicle infusion, the electrical activity (Fig. 3A; from top to bottom) in the dome, upper bladder, lower bladder, base of the bladder, and EUS showed a clear association with the generation of IPHFOs (bottom panel). The intravesical application of AF-353 in the same animal generated a clear reduction of electrical signals in the corresponding bladder regions (Fig. 3B). Pharmacological inhibition of P2X3R led to an increase in the intercontractile interval (Fig. 4A) and a decrease in both the bladder pressure threshold (Fig. 4B) and IPHFO duration (Fig. 4C), reproducing cystometric effects seen in previous studies [20].

\section{Associations Between LUT Electrical Signals and Bladder Pressure}

We distinguished 3 different stages of electrical signal generation during the voiding event (Fig. 5A). For clarity, only the signals for the dome (blue line), the EUS (black line), and bladder pressure (red line) are shown in this example. The 3 different stages corresponded to increased electrical activity prevoid (Fig. 5B), electrical activity during the actual voiding event (Fig. 5C), and postvoid electrical activity (Fig. 5D). The electrical activity in all channels returned to baseline levels after the end of the IPHFO and remained at baseline until a subsequent voiding event was initiated (Fig. 2).

\section{Intravesical Inhibition of P2X3R Decreased Electrical Signals in the Bladder, but Not the EUS}

Intravesical application of the P2X3R antagonist (Fig. 6) led to an appreciable reduction in electrical signals from the bladder (blue line for dome) compared to those from the EUS (black line) during the IPHFO (red line). Although a trend was observed in the prevoid region (Fig. 6B), the main effects were recorded in the bladder signals during the actual voiding event (Fig. 6C). The electrical activity from the EUS was not affected during any portion of the voiding or postvoid phases (Fig. 6D). As observed with the vehicle, at the end of the IPHFO, the electrical activity returned to baseline levels.

\section{Intravesical Antagonism of P2X3R Decreased the Amplitude of Electrical Signals in the LUT}

Electrocardiograms showed no difference in the QRS amplitude (Fig. 7A) in the prevoid, void, or postvoid stages when saline (clear bars) or AF-353 (black bars) was applied intravesically. There was a trend towards amplitude reduction in the 


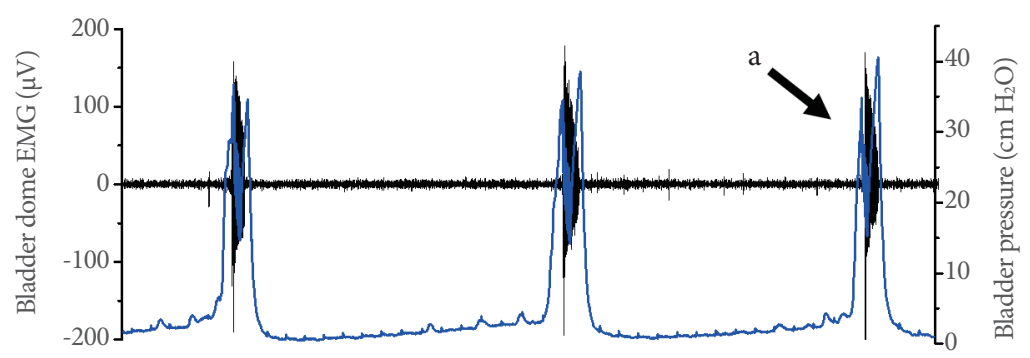

A
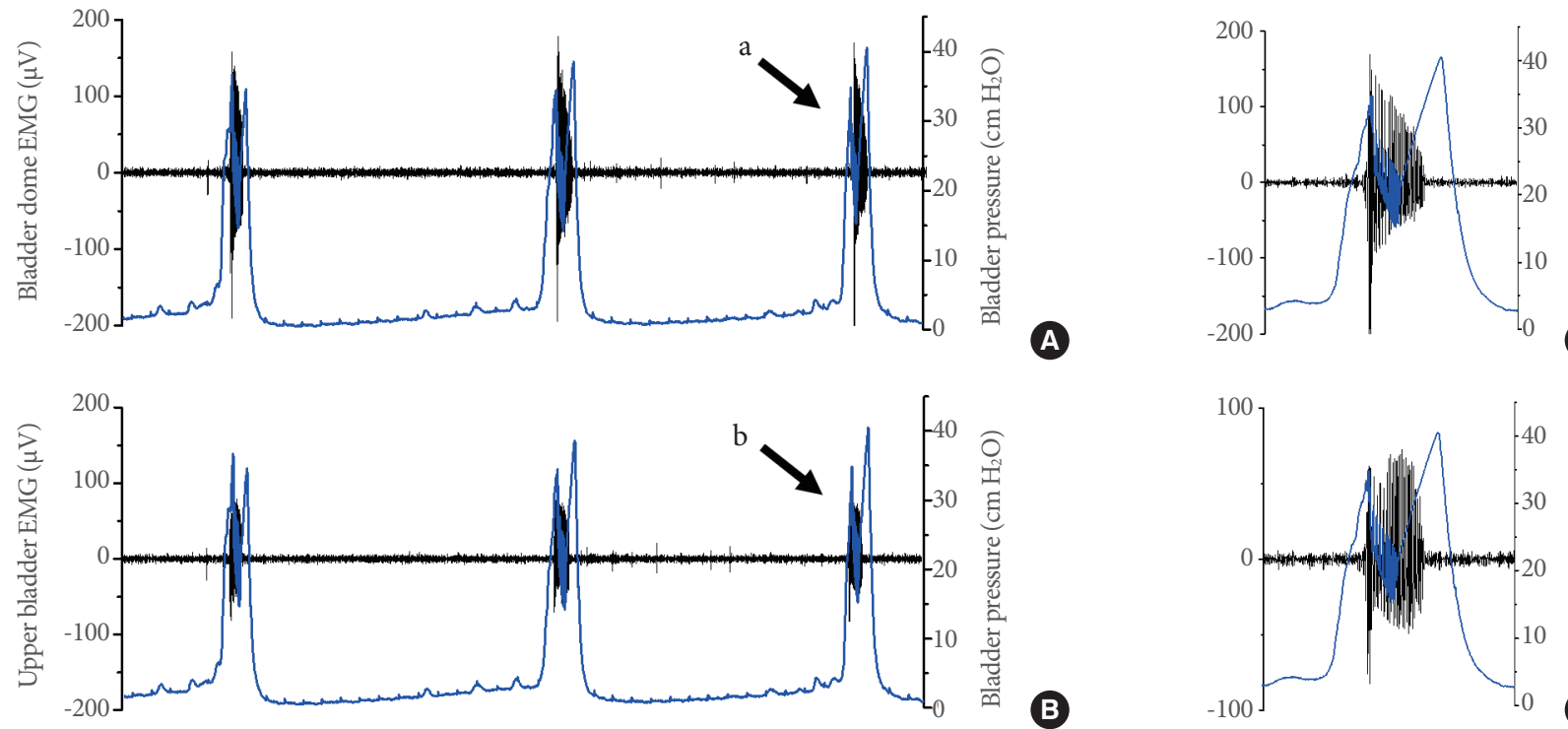

B

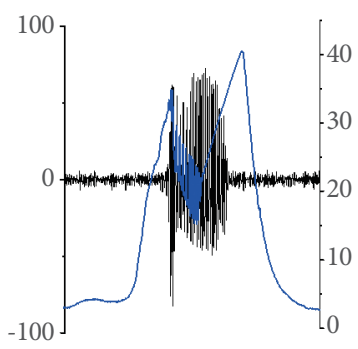

(อ

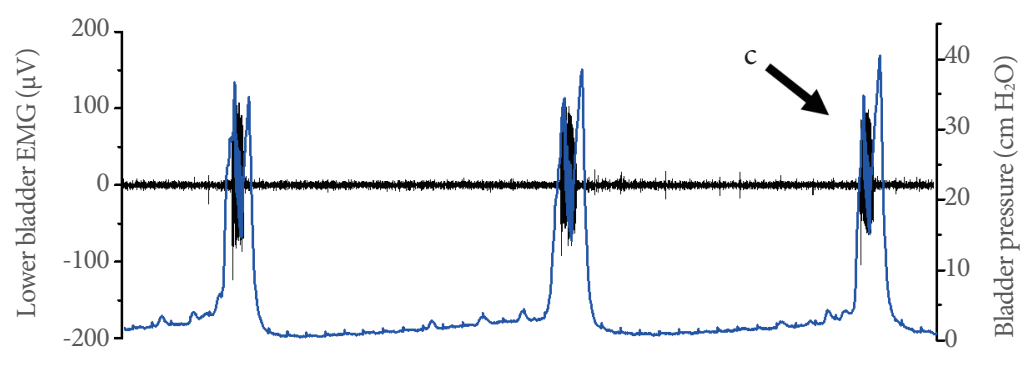

$\boldsymbol{0}$
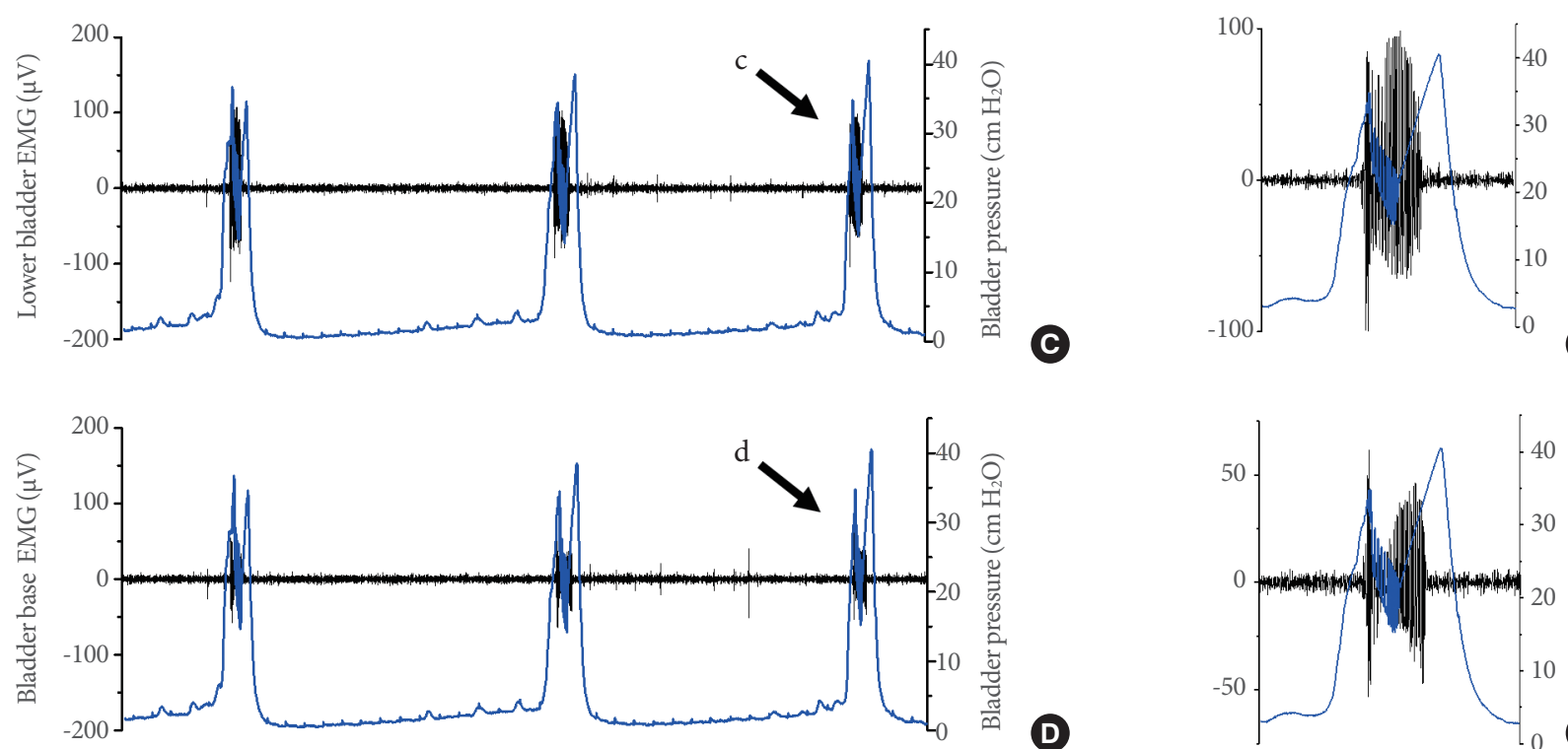

(D)
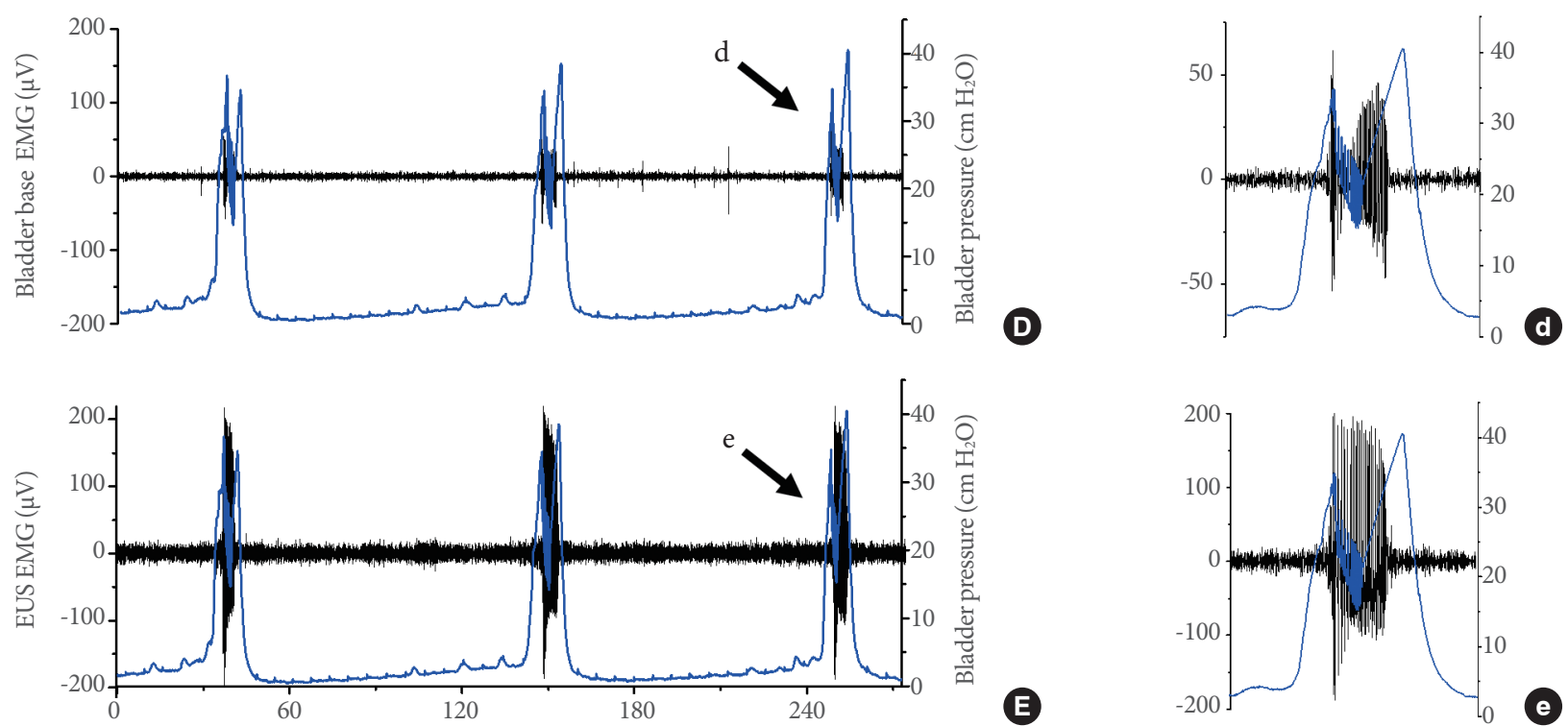

B

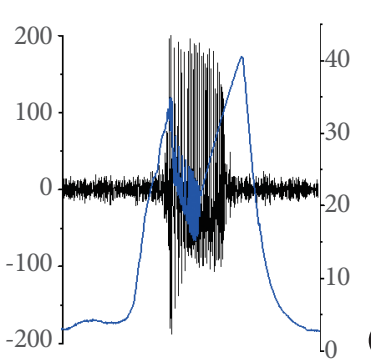

Fig. 2. Electrical activity at different levels of the lower urinary tract. The figure shows changes in electromyography (EMG) responses (dark lines) and bladder pressure (blue lines) at the bladder dome (A), upper bladder (B), lower bladder (C), bladder base (D), and external urethral sphincter (EUS) (E) during 3 filling/voiding cycles. The left panels show an amplified view of the contraction indicated on each panel (a-e). Note the differences in the amplitude of the EMG response as well as the simultaneous occurrence of EMG signals with intraluminal pressure high-frequency oscillations in panels a-e. 

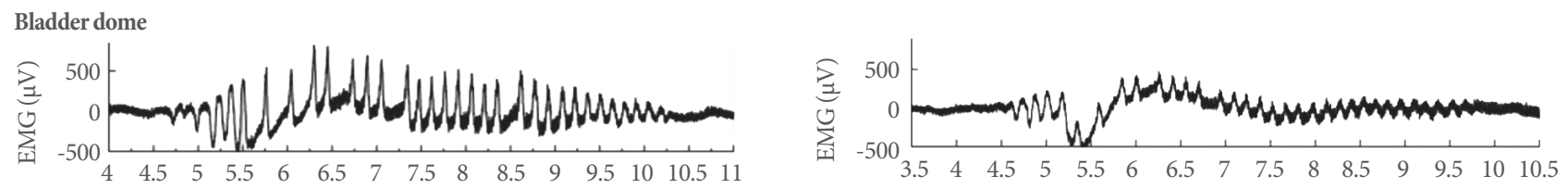

Upper bladder
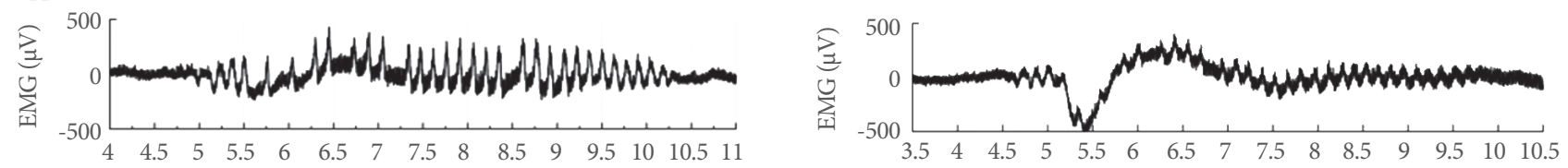

Lower bladder
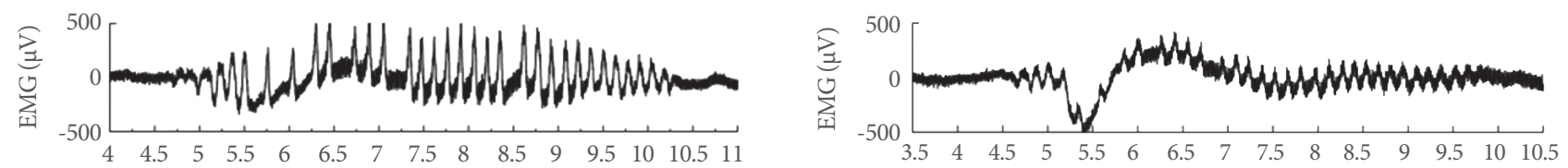

Bladder base
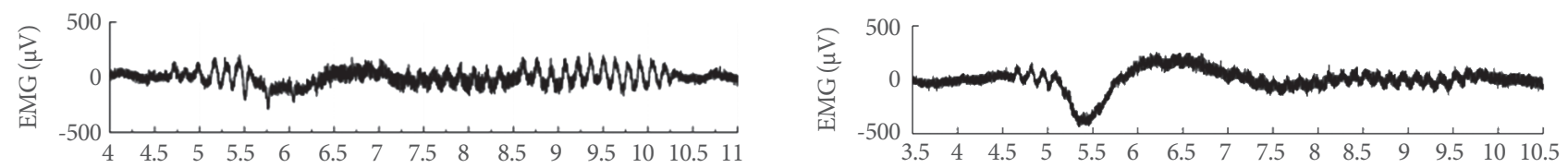

EUS
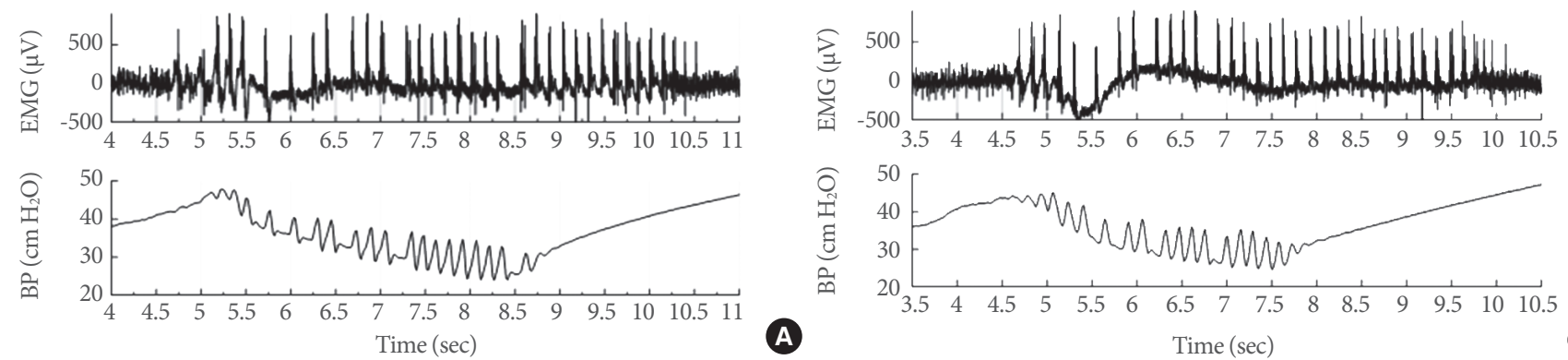

Fig. 3. Electrical signals in the lower urinary tract (LUT) and the effects of P2X3-containing receptor (P2X3R) inhibition. Electrical responses at different levels of the LUT during intravesical perfusion of vehicle (A) or the specific P2X3R antagonist AF-353 at a concentration of $10 \mu \mathrm{M}(\mathrm{B})$. In both examples, the panels represent electrical activity, from top to bottom, in the bladder dome, upper bladder, lower bladder, bladder base, and EUS. The bottom panel shows the corresponding changes in intravesical pressure and intraluminal pressure high-frequency oscillations during an effective micturition event. EMG, electromyography; BP, bladder pressure.
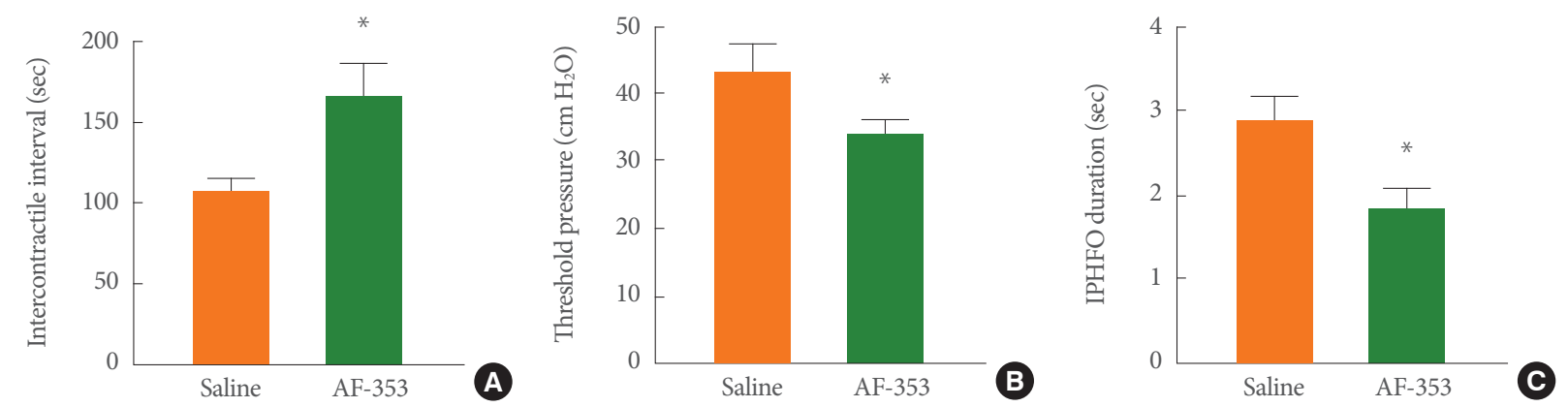

Fig. 4. Effects of AF-353 on cystometric parameters during electromyographic characterization of the lower urinary tract. (A) The intercontractile interval between voiding events was extended by the intravesical inhibition of P2X3-containing receptors with AF-353. (B) The threshold pressure before the triggering of a voiding contraction was reduced with AF-353. (C) The duration of intraluminal pressure high-frequency oscillations was also reduced. IPHFO, intraluminal pressure high frequency oscillation. Unpaired Student ttest. ${ }^{*} \mathrm{P}<0.05$ versus saline infusion. 


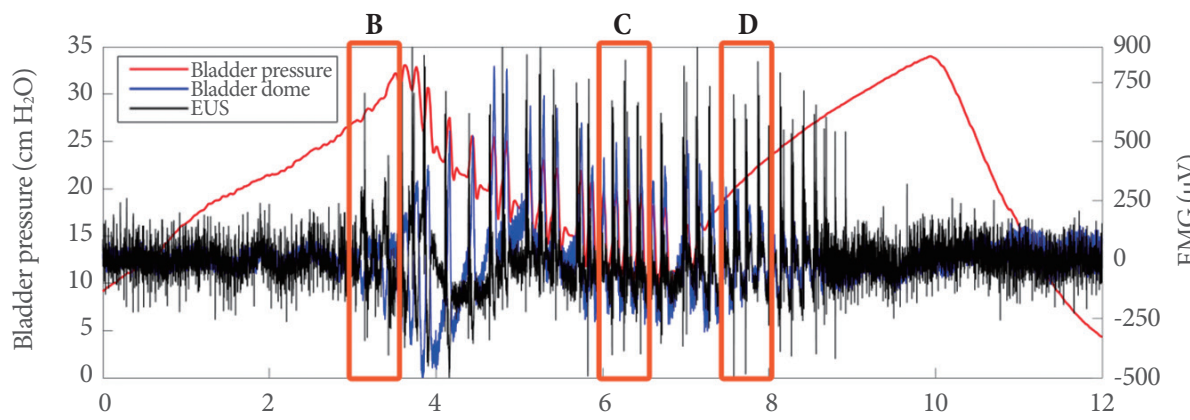

A

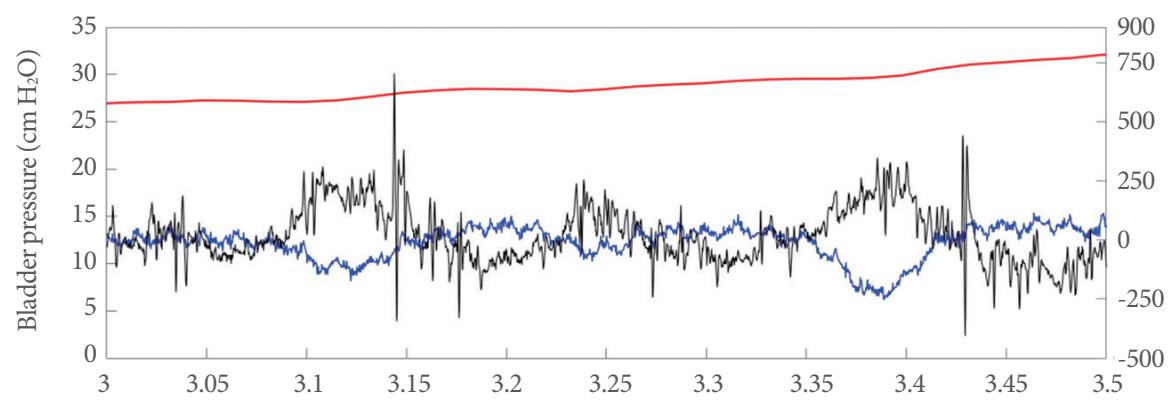

B

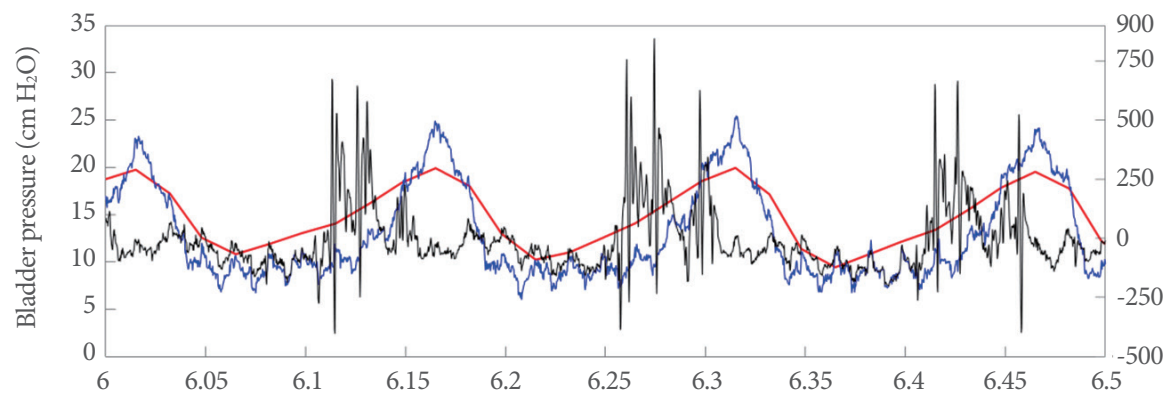

C

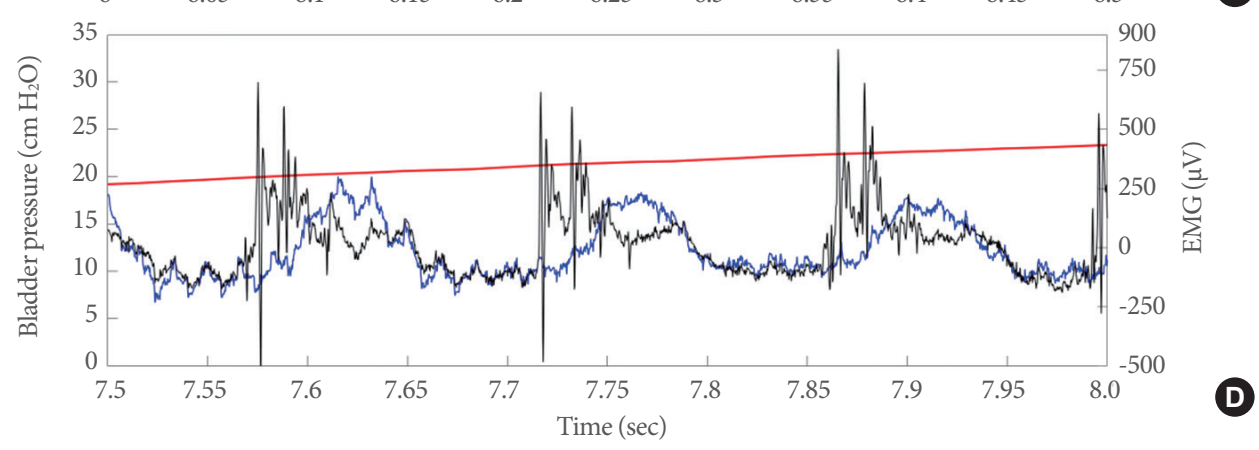

Fig. 5. The electrical responses in the lower urinary tract during open cystometry included 3 different phases. (A) Superimposed electrical signals from the bladder dome (blue line) and external urethral sphincter (EUS) (black line), as well as bladder pressure (red line) during a full voiding event. Superimposed electrical signals from the bladder dome and EUS during the prevoid stage (B), during the actual voiding event $(\mathrm{C})$, and during the postvoid stage (D). EMG, electromyography.

electrical signals of the LUT during the pre- and postvoid stages after P2X3R inhibition. Nonetheless, the amplitudes of the electrical signals during IPHFO were significantly reduced by the intravesical application of AF-353 at the bladder dome (Fig. 7B), the upper bladder region (Fig. 7C), and the lower bladder region (Fig. 7D). The amplitude of the electrical signals in the bladder base or in the EUS did not change after P2X3R antagonist administration (Fig. 7E, F).

\section{DISCUSSION}

We made simultaneous recordings of the electrical activity gen- 


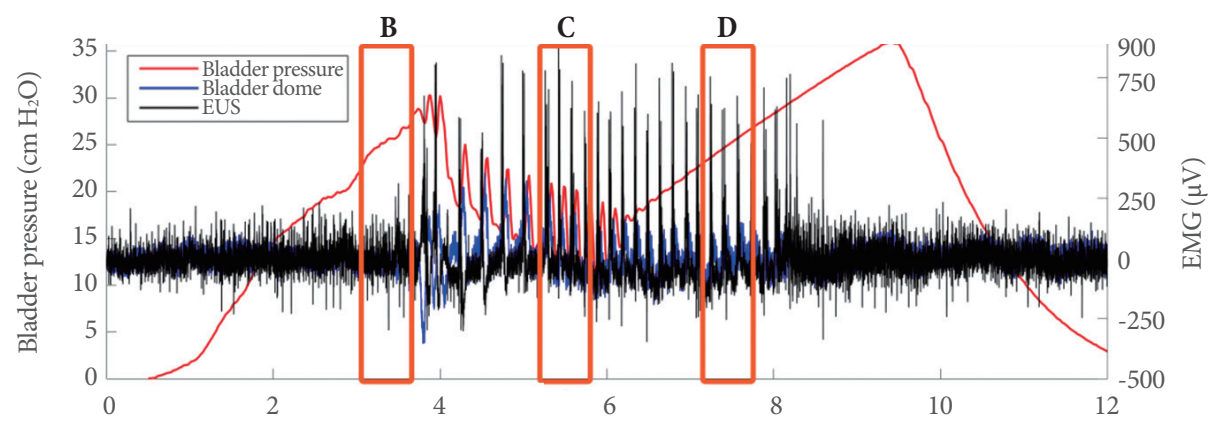

(A)

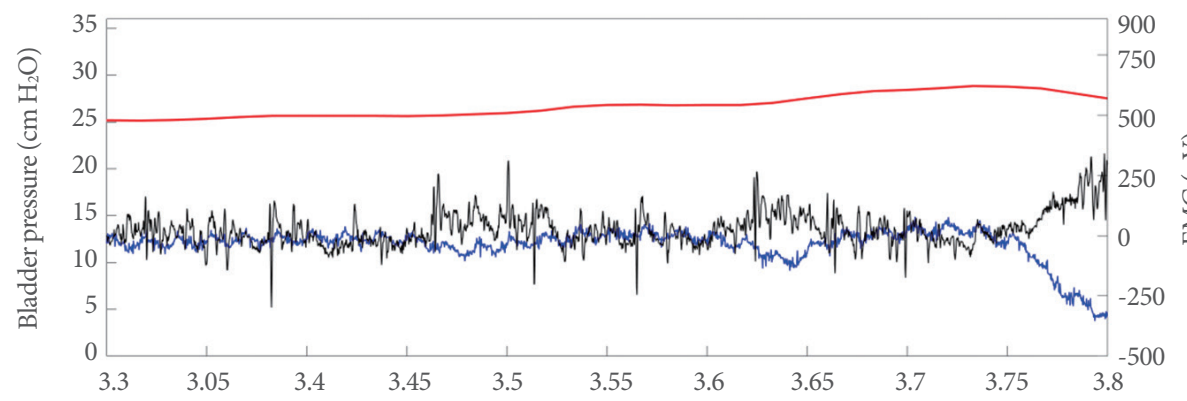

B

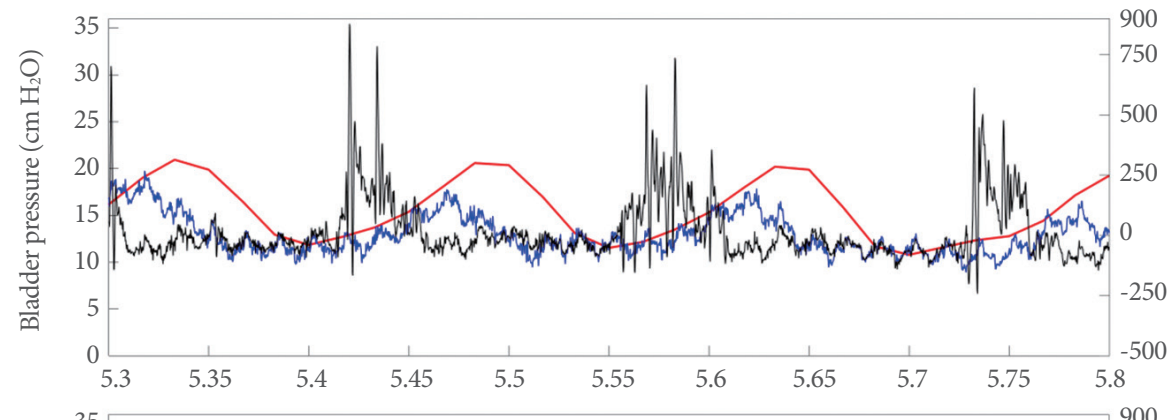

C

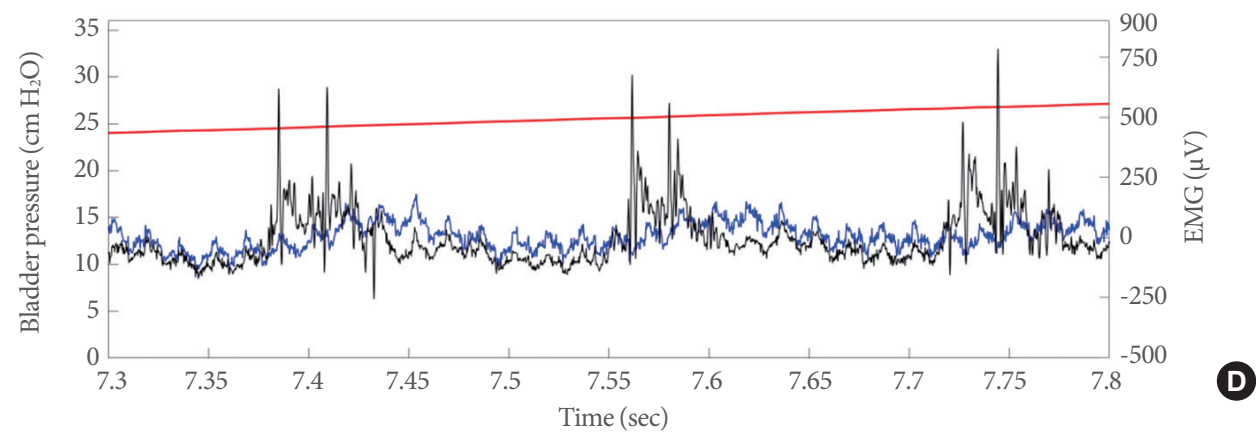

Fig. 6. Intravesical inhibition of P2X3-containing receptors (P2X3R) attenuated the electrical responses in the lower urinary tract. (A) Representative example of superimposed electrical signals from the bladder dome (blue line) and external urethral sphincter (EUS) (black line), as well as bladder pressure (red line) during a full voiding event during P2X3R inhibition by the intravesical perfusion of $10 \mu \mathrm{M}$ AF-353. Superimposed electrical signals from the bladder dome and EUS during the prevoid stage (B), during the actual voiding event (C), and during the postvoid stage (D). EMG, electromyography.

erated in various regions of the LUT during open cystometry in anesthetized female rats. Due to the geometry and flexibility of the electrodes, no perforations of the bladder occurred during cystometric filling, providing a greater contact area for signal acquisition than can be accomplished using standard elec- trodes. As has been observed during electromyographic recordings from the EUS made using needle electrodes [16,23], we noted that electrical activity started before voiding. Interestingly, when bladder pressure and electrical waveforms were superimposed, a consistent trend was observed in which the dome of 

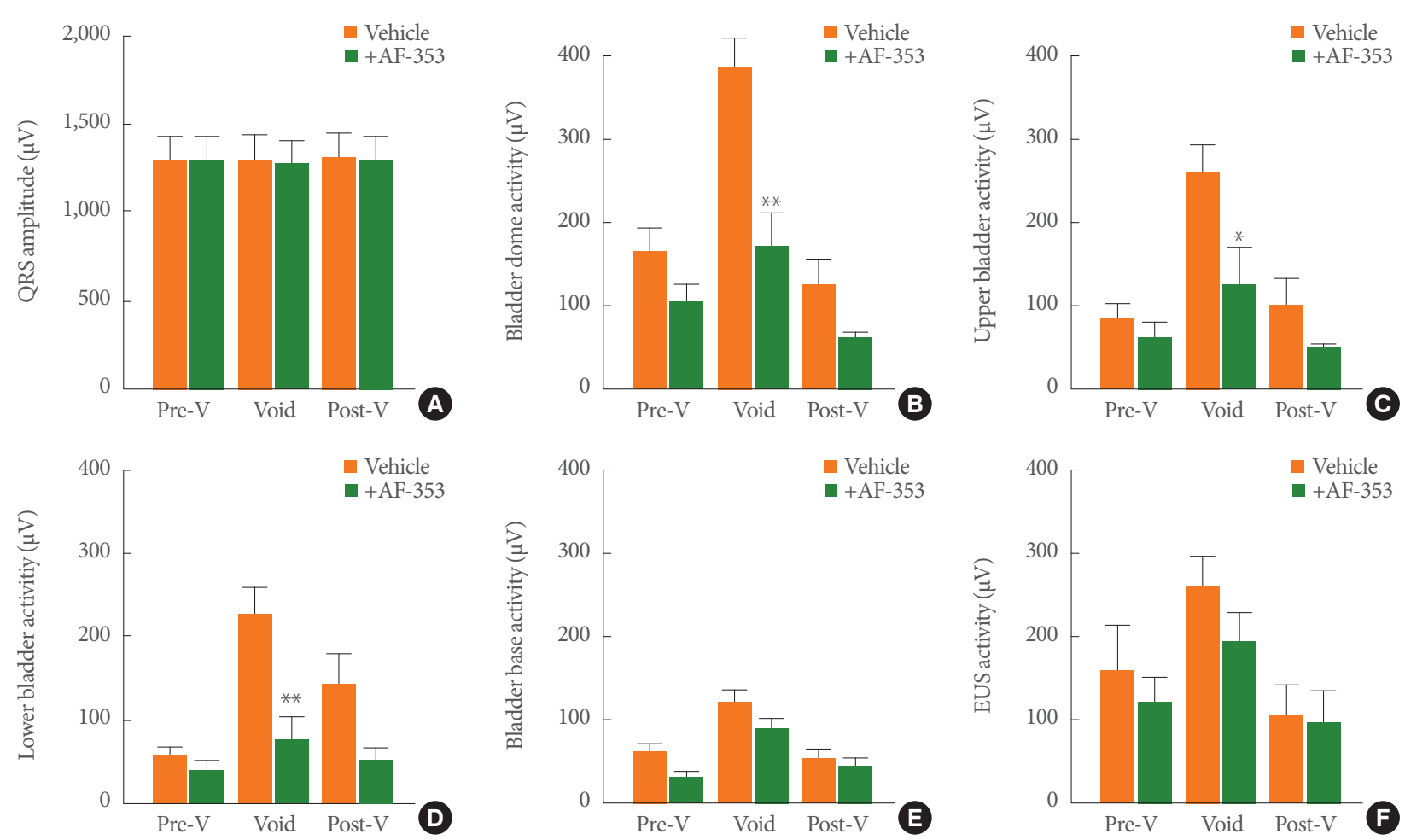

Fig. 7. Group analysis of electrical signals and the effect produced by intravesical inhibition of P2X3-containing receptors. (A) Amplitude of the QRS electrocardiogram response at different stages of the voiding events during the intravesical infusion of vehicle or 10 $\mu \mathrm{M}$ AF-353. Amplitudes of electrical signals at the bladder dome (B), upper bladder (C), lower bladder (D), bladder base (E), and external urethral sphincter $(\mathrm{F})$. Values represent the mean \pm standard error of the mean for 8 rats. ${ }^{*} \mathrm{P}<0.05$ versus the corresponding voiding stage and lower urinary tract (LUT) region. ${ }^{* *} \mathrm{P}<0.01$ versus the corresponding voiding stage and LUT region. Pre-V, prevoid; Post-V, postvoid.

the bladder was consistently $180^{\circ}$ out of phase with the EUS (Fig. 3B). Although attenuated, this activity pattern from the detrusor muscle also occurred during voiding and in the presence of AF-353, supporting the idea that during bladder distension, P2X3R activation is required for an effective voiding contraction to be generated [24].

EUS relaxation and detrusor contraction must be coordinated for an efficient voiding event to occur [25]. Changes in LUT bladder pressure and electromyographic activity throughout typical voiding events allowed us to evaluate the collaborative efforts of multiple systems during the micturition process. Further, the effects of the intravesical application of a P2X3R antagonist support the key role of P2X3R in bladder sensory communication and suggest that they are required for the initiation of an efficient micturition reflex.

The ATP released from nerve terminals or urothelial cells can trigger the activation of $\mathrm{P} 2 \mathrm{X} 1$ receptors expressed in detrusor cells and then stimulate bladder contractions [26]. Further- more, those changes in ATP also activate P2X3R signals that convey information regarding bladder-filling conditions for pain perception or initiation of the micturition reflex [27]. The results of this study demonstrate the regulatory role played by intravesical P2X3R. Specifically, we were able to elucidate an orderly pattern of activity within each voiding cycle and to observe attenuation resulting from P2X3R inhibition.

In a clinical environment, muscarinic antagonists are used to manage neurogenic bladder overactivity and other dysfunctions associated with disorders such as diabetes, spinal cord injuries, multiple sclerosis, or other neurological conditions $[28,29]$. These antimuscarinic therapies are accompanied by secondary side effects that contribute to low compliance by patients, motivating the search for alternative treatments. With this in mind, P2X3R inhibition via the intravesical infusion of AF-353 during open cystometry may be a pharmacological candidate for attenuating bladder overactivity. Although we did not evaluate the permeability of AF-353 into different bladder 
layers, due to the presence of DMSO and the observed decrease in the strength of the electrical signals, we can assume that it does penetrate the layers of the bladder, reaching the afferent innervation. Finally, the intravesical inhibition of P2X3R by a low concentration of AF-353 [20] supports the role of these receptors in regulating the activity of bladder afferent nerves, and conceivably the urothelial sensory role of these receptors $[3,20,30]$.

The electrophysiological measurements during voiding contractions suggest that electromyographic activity begins before, peaks at, and ends after the IPHFO recedes. These phases were observed during saline infusion and intravesical AF-353 infusion, although they were attenuated with the latter. This trend was clearly defined and therefore suggestive of a signal activation pattern that is transmitted throughout the detrusor regions of the bladder. There is a dearth in detrusor musculature activity during the intercontractile interval and bladder-filling (Fig. 2), illustrating the absence of any appreciable detrusor electrical activity throughout the filling phase. Leading up to the voiding phase, the bladder seems to remain in this passive state until a nociceptive response and the recruitment of mechanosensitive receptors, including P2X3R, help initiate the voiding phase [2]. These characteristics of amplitude, phased signal generation, and quiescent detrusor electrical activity despite bladder movement due to filling help eliminate the possibility that our recordings were mere signals produced during muscle contractions.

Additionally, the amplitudes of the electrical waves were significantly reduced during the intravesical inhibition of P2X3R. It has been shown that efficient bladder contractions involve neural and smooth muscle components [2,31], and based on the electrical properties of the detrusor and its sensitivity to afferent inhibition, we propose that the origin of the LUT activity recordings was likely neurogenic, with a strong secondary myogenic component. Interestingly, our analysis suggests that electrical signals between the bladder dome and the EUS were nearly $180^{\circ}$ out of synchrony during both saline and AF-353 infusion. This observation suggests the presence of a coordinated action between the relaxation of the urethra and the contractions of the detrusor to generate IPHFOs, which are required for efficient voiding in anesthetized female rats.

We observed that the dome of the bladder showed the largest wave amplitude, followed by the EUS, upper bladder, lower bladder, and the bladder base. These observations could be suggestive of a pattern of contractile propagation with regard to the amplitude of the electrical response and the initiation of mictu- rition based on the type of innervation at a particular region of the LUT. For example, parasympathetic innervation coreleasing acetylcholine and ATP in the dome may be different (i.e., higher) than in the bladder base or the EUS, thus explaining the reduction in amplitude generated by the P2X3R antagonist. This trend was observed when AF-353 was applied, albeit with significantly reduced wave amplitude, but these findings are consistent with the proposal that the signals may be primarily neurogenic in origin.

The basic suggestion generated by the findings of our study is that attenuation of the P2X3-component of the afferent signal may produce a reduced contractile force because the animal may not be able to sense the actual bladder volume (i.e., the electromyographic and efferent responses are weaker). This sensory 'impairment' may be due to the inhibition of $\mathrm{P} 2 \mathrm{X} 3 \mathrm{R}$ in afferent nerve terminals, urothelial cells, or both. In the latter case, urothelial P2X3R may be acting as an amplifier for the release of ATP for assisting afferent activation. Finally, the correlation between urinary bladder distention and mechanical reflex capacity may be diminished due to the attenuated P2X3Rmediated signal. An exploration of simultaneous detrusor and EUS electrophysiology may lead to a better understanding of experimental models of the LUT in health and disease.

\section{ACKNOWLEDGEMENTS}

The authors wish to thank Dr. Anthony P. Ford from Afferent Pharmaceuticals (Now part of Merck \& Co., Inc.) for the donation of AF-353.

\section{REFERENCES}

1. Fowler CJ, Griffiths D, de Groat WC. The neural control of micturition. Nat Rev Neurosci 2008;9:453-66.

2. Merrill L, Gonzalez EJ, Girard BM, Vizzard MA. Receptors, channels, and signalling in the urothelial sensory system in the bladder. Nat Rev Urol 2016;13:193-204.

3. Birder L, Andersson KE. Urothelial signaling. Physiol Rev 2013; 93:653-80.

4. Heppner TJ, Werner ME, Nausch B, Vial C, Evans RJ, Nelson MT. Nerve-evoked purinergic signalling suppresses action potentials, $\mathrm{Ca} 2+$ flashes and contractility evoked by muscarinic receptor activation in mouse urinary bladder smooth muscle. J Physiol 2009; 587(Pt 21):5275-88.

5. Cockayne DA, Hamilton SG, Zhu QM, Dunn PM, Zhong Y, Nova- 
kovic S, et al. Urinary bladder hyporeflexia and reduced pain-related behaviour in P2X3-deficient mice. Nature 2000;407:1011-5.

6. Takezawa K, Kondo M, Kiuchi H, Ueda N, Soda T, Fukuhara S, Takao T, et al. Authentic role of ATP signaling in micturition reflex. Sci Rep 2016;6:19585.

7. Burnstock G. Purinergic signalling in the urinary tract in health and disease. Purinergic Signal 2014;10:103-55.

8. Cruz Y, Downie JW. Abdominal muscle activity during voiding in female rats with normal or irritated bladder. Am J Physiol Regul Integr Comp Physiol 2006;290:R1436-45.

9. Cruz Y, Pastelín C, Balog BM, Zaszczurynski PJ, Damaser MS. Somatomotor and sensory urethral control of micturition in female rats. Am J Physiol Renal Physiol 2014;307:F1207-14.

10. D’Amico SC, Collins WF 3rd. External urethral sphincter motor unit recruitment patterns during micturition in the spinally intact and transected adult rat. J Neurophysiol 2012;108:2554-67.

11. D'Amico SC, Schuster IP, Collins WF 3rd. Quantification of external urethral sphincter and bladder activity during micturition in the intact and spinally transected adult rat. Exp Neurol 2011; 228:59-68.

12. Pastelín CF, Juárez R, Damaser MS, Cruz Y. Neural pathways of somatic and visceral reflexes of the external urethral sphincter in female rats. J Comp Neurol 2012;520:3120-34.

13. Kakizaki H, Fraser MO, De Groat WC. Reflex pathways controlling urethral striated and smooth muscle function in the male rat. Am J Physiol 1997;272(5 Pt 2):R1647-56.

14. Schneider MP, Hughes FM Jr, Engmann AK, Purves JT, Kasper H, Tedaldi M, et al. A novel urodynamic model for lower urinary tract assessment in awake rats. BJU Int 2015;115 Suppl 6:8-15.

15. Kadekawa K, Yoshimura N, Majima T, Wada N, Shimizu T, Birder LA, et al. Characterization of bladder and external urethral activity in mice with or without spinal cord injury--a comparison study with rats. Am J Physiol Regul Integr Comp Physiol 2016;310:R7528.

16. LaPallo BK, Wolpaw JR, Chen XY, Carp JS. Contribution of the external urethral sphincter to urinary void size in unanesthetized unrestrained rats. Neurourol Urodyn 2016;35:696-702.

17. Gever JR, Soto R, Henningsen RA, Martin RS, Hackos DH, Panicker S, et al. AF-353, a novel, potent and orally bioavailable P2X3/ P2X2/3 receptor antagonist. Br J Pharmacol 2010;160:1387-98.

18. Munoz A, Somogyi GT, Boone TB, Ford AP, Smith CP. Modula- tion of bladder afferent signals in normal and spinal cord-injured rats by purinergic $\mathrm{P} 2 \mathrm{X} 3$ and $\mathrm{P} 2 \mathrm{X} 2 / 3$ receptors. BJU Int 2012;110(8 Pt B):E409-14.

19. Ford AP. In pursuit of $\mathrm{P} 2 \mathrm{X} 3$ antagonists: novel therapeutics for chronic pain and afferent sensitization. Purinergic Signal 2012; 8(Suppl 1):3-26.

20. Ferguson AC, Sutton BW, Boone TB, Ford AP, Munoz A. Inhibition of urothelial $\mathrm{P} 2 \mathrm{X} 3$ receptors prevents desensitization of purinergic detrusor contractions in the rat bladder. BJU Int 2015;116: 293-301.

21. Andersson KE, Soler R, Füllhase C. Rodent models for urodynamic investigation. Neurourol Urodyn 2011;30:636-46.

22. National Research Council (U.S.), Committee for the Update of the Guide for the Care and Use of Laboratory Animals; Institute for Laboratory Animal Research (U.S.). Guide for the care and use of laboratory animals. 8th ed. Washington, DC: National Academies Press; 2011.

23. Juárez R, Zempoalteca R, Pacheco P, Lucio RA, Medel A, Cruz Y. Activity of the external urethral sphincter evoked by genital stimulation in male rats. Neurourol Urodyn 2016;35:914-919.

24. Andersson KE. Purinergic signalling in the urinary bladder. Auton Neurosci 2015;191:78-81.

25. Langdale CL, Grill WM. Phasic activation of the external urethral sphincter increases voiding efficiency in the rat and the cat. Exp Neurol 2016;285(Pt B):173-81.

26. North RA, Jarvis MF. P2X receptors as drug targets. Mol Pharmacol 2013;83:759-69.

27. Burnstock G. Purinergic signalling in the lower urinary tract. Acta Physiol (Oxf) 2013;207:40-52.

28. Panicker JN, Fowler CJ, Kessler TM. Lower urinary tract dysfunction in the neurological patient: clinical assessment and management. Lancet Neurol 2015;14:720-32.

29. Abrams P, Andersson KE. Muscarinic receptor antagonists for overactive bladder. BJU Int 2007;100:987-1006.

30. Munoz A, Yazdi IK, Tang X, Rivera C, Taghipour N, Grossman $\mathrm{RG}$, et al. Localized inhibition of P2X7R at the spinal cord injury site improves neurogenic bladder dysfunction by decreasing urothelial P2X3R expression in rats. Life Sci 2017;171:60-67.

31. de Groat WC, Griffiths D, Yoshimura N. Neural control of the lower urinary tract. Compr Physiol 2015;5:327-96. 